Supporting Information

\title{
Sensible Functional Linear Discriminant Analysis Effectively Discriminates Enhanced Raman Spectra of Mycobacterium Species
}

Wei-Chih Cheng ${ }^{1}$, Lu-Hung Chen ${ }^{2, *}$, Ci-Ren Jiang ${ }^{3, *}$, Yu-Ming Deng ${ }^{4}$, Da-Wei Wang ${ }^{5}$, Chi-Hung $\operatorname{Lin}^{6}$, Ruwen Jou ${ }^{4,6,7 *}$, Juen-Kai Wang ${ }^{1,8 *}$, and Yuh-Lin Wang ${ }^{1,9 *}$

${ }^{1}$ Institute of Atomic and Molecular Sciences, Academia Sinica, Taipei, Taiwan

${ }^{2}$ Department of Applied Mathematics, National Chung Hsing University, Taichung, Taiwan

${ }^{3}$ Institute of Statistical Science, Academia Sinica, Taipei, Taiwan

${ }^{4}$ Reference Laboratory of Mycobacteriology, Centers for Disease Control, Taipei, Taiwan

5Institute of Information Science, Academia Sinica, Taipei, Taiwan

${ }^{6}$ Institute of Microbiology and Immunology, National Yang Ming University, Taipei, Taiwan

${ }^{7}$ Tuberculosis Research Center, Centers for Disease Control, Taipei, Taiwan

${ }^{8}$ Center for Condensed Matter Sciences, National Taiwan University, Taipei, Taiwan

${ }^{9}$ Department of Physics, National Taiwan University, Taipei, Taiwan

\section{Corresponding Authors}

*E-mail: luhung@email.nchu.edu.tw; cirenjiang@stat.sinica.edu.tw; rwj@cdc.gov.tw; jkwang@ntu.edu.tw; ylwang@pub.iams.sinica.edu.tw 
Section S1. List of Mycobacterium species studied in this work

Table S1. Mycobacterium species studied in this work

\begin{tabular}{|c|c|c|}
\hline \multirow{7}{*}{ Reference strains } & \multirow{2}{*}{ Mycobacterium tuberculosis } & ATCC 25177 \\
\hline & & ATCC 27294 \\
\hline & Mycobacterium microti & ATCC 35782 \\
\hline & Mycobacterium peregrinum & ATCC 700686 \\
\hline & \multirow{2}{*}{ Mycobacterium intracellulare } & ATCC 35763 \\
\hline & & ATCC 35761 \\
\hline & $\begin{array}{l}\text { Mycobacterium avium } \\
\text { subspecies paratuberculosis }\end{array}$ & ATCC 49164 \\
\hline \multirow{12}{*}{ Clinical isolates } & \multirow{4}{*}{ Mycobacterium tuberculosis } & CAP 110200454 \\
\hline & & CAP 110200456 \\
\hline & & CAP 120201278 \\
\hline & & CAP 120206862 \\
\hline & Mycobacterium abscessus & CAP 110200452 \\
\hline & Mycobacterium intracellulare & CAP 110200453 \\
\hline & Mycobacterium gordonae & CAP 110200455 \\
\hline & Mycobacterium marinum & CAP 120201275 \\
\hline & Mycobacterium mucogenicum & CAP 120201276 \\
\hline & Mycobacterium chelonae & CAP 120201279 \\
\hline & Mycobacterium neoaurum & CAP 120206859 \\
\hline & Mycobacterium xenopi & CAP 120206861 \\
\hline
\end{tabular}




\section{Section S2. Structural and optical characterizations of AgNP/AAO substrate and its SERS performance of adenine}

Scanning electron microscopy was used to determine the diameter and the packing configuration of the Ag nanoparticles of AgNP/AAO substrate, while cross-section transmission electron microscopy was employed to examine the interparticle spacing between adjacent nanoparticles. ${ }^{1}$ The average particle diameter is $45 \mathrm{~nm}$ and the mean interparticle gap is $5 \mathrm{~nm}$. The plasmon resonance wavelength as a function of the interparticle spacing was investigated with far-field scattering spectroscopy. ${ }^{2}$ The scattering spectrum exhibited a prominent peak at $550 \mathrm{~nm}$ with a full width at maximum of $\sim 150 \mathrm{~nm}$. Accordingly, both the excitation wavelength at $632.8 \mathrm{~nm}$ and the Stokes radiation wavelength ranging from 650 to $720 \mathrm{~nm}$ are covered by this plasmon resonance to enable Raman enhancement. The scattering feature was examined across the substrate surface to reveal its uniformity. The resonance wavelength was found to vary by less than $10 \mathrm{~nm}$, suggesting that the electromagnetic enhancement factor of the SERS effect is uniform across the substrate. This finding is consistent with the fact that the SERS signal strength is varied by $<10 \%$ over the substrate surface. Scattering-type scanning nearfield optical microcopy with a laterial resolution fo $\sim 5 \mathrm{~nm}$ and a vertical resolution of $<10 \mathrm{~nm}$ was used to reveal the local field distribution (or specifically the hot spots) of the substrate. ${ }^{3}$ Lastly, Figure S1 shows the concentration curve of the SERS signal strength of adenine at $730 \mathrm{~nm}$. It exhibits a Langmuir-type shape, ${ }^{4}$ indicating a basic adsorption-desorption behavior of adenine on the substrate surface. 


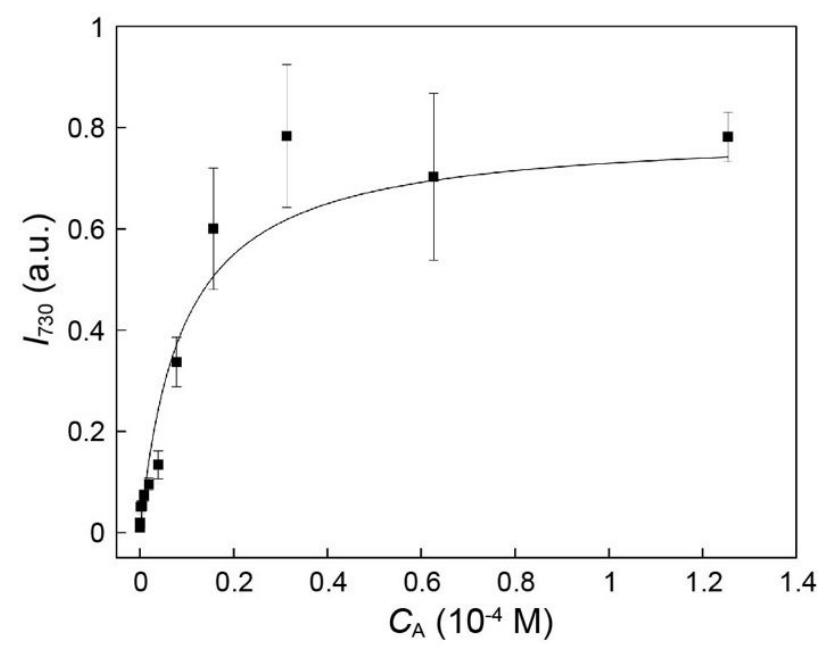

Figure S1. Integrated SERS signal of $730-\mathrm{cm}^{-1}$ peak of adenine $\left(I_{730}\right)$ vs. adenine concentration $\left(C_{\mathrm{A}}\right)$. The solid line is the fitted curve of Langmuir sorption-isotherm $\operatorname{model}^{4}-I_{730}=I_{730}^{\infty}$. $\left(L \cdot C_{\mathrm{A}}\right) /\left(1+L \cdot C_{\mathrm{A}}\right)$ —obtained an affinity of $L=1.1 \times 10^{5} \mathrm{M}^{-1} \cdot I_{730}^{\infty}$ is $I_{730}$ at infinite concentration. 


\section{Section S3. Mathematical description of SLDA method}

Linear discriminant analysis (LDA) is commonly employed for classification in different fields. Briefly, LDA aims to identify a few projections that maximize the projected distance between class centers while simultaneously minimizes the projected distance within the same class and performs classification in the projected subspace. The LDA projections $b$ are often obtained through a generalized eigen-equation, $\eta b=\Sigma_{W}^{-1} \Sigma_{B} b$, where $\Sigma_{W}^{-1}$ is the inverse of the within-class covariance matrix, $\Sigma_{B}$ is the between-class covariance matrix and $\eta \geq 0$ is the generalized eigenvalue. However, when the data dimension is large, $\Sigma_{W}^{-1}$ is either unstable or non-existent. Therefore, some dimension reduction approaches are applied before LDA is performed, and it is crucial to select a proper one. Take principal component analysis (PCA) for example. Without considering any class information, PCA reduces data dimension through projecting the data to a smaller subspace that is spanned by the eigenvectors (of $\Sigma_{W}$ ) corresponding to a few relatively large eigenvalues, and those eigenvectors corresponding relatively small eigenvalues are truncated. However, when the subtle but useful information for classification exists in the truncated eigenvectors, PCA simply worsens the results. To avoid such a circumstance in the dimension reduction step, sensible functional linear discriminant analysis (SLDA) $)^{5}$ was proposed to tackle the non-invertibility issue of a covariance operator while extending LDA to classify functional data in $L_{2}$. Specifically, to avoid inverting a non-invertible covariance operator, SLDA searches the optimal LDA projections in two orthogonal subspaces, $S_{\perp}$ and $S_{\|}$(defined below), sequentially; in particular, LDA merely searches projections in $S_{\|}$, but the projections in $S_{\perp}$ lead to nearly perfect classification. Suppose the dataset consists of $c$ classes (or species in this paper). Let $I_{j}(v)$ be an $L_{2}$ stochastic process in class $j$ with mean function $\mu_{j}(v)$ and covariance function $\Gamma_{w}(u, v)=\operatorname{cov}\left(I_{j}(u), I_{j}(v)\right)$. The covariance function $\Gamma_{w}$ is assumed to be the same for all classes and is also called the within-class covariance function. On the other hand, the between-class covariance function is defined as $\Gamma_{B}(u, v)=\sum_{k=1}^{c} \pi_{k}\left\{\mu_{k}(u)-\mu(u)\right\}\left\{\mu_{k}(v)-\mu(v)\right\}$, where $\pi_{k}$ is the probability that a randomly selected function $I(v)$ is in class $k$ and $\mu(v)=$ 
$\sum_{k=1}^{c} \pi_{k} \mu_{k}(v)$ is the overall mean function. For convenience, let $S_{B}$ (resp. $S_{W}$ ) be the eigen-space of $\Gamma_{B}\left(\right.$ resp. $\left.\Gamma_{W}\right)$ and denote the inner product in $L_{2}$ as $\langle a, b\rangle=\int a(v) b(v) d v$ and $\Gamma b=\int \Gamma(u, v) b(v) d v$. Mathematically, SLDA is defined as sequentially finding the projections $\left\{\beta_{j}\right\}_{j=1}^{c^{\prime}+c^{\prime \prime}}$ :

and

$$
\beta_{1}=\underset{\beta \in S_{\perp}}{\operatorname{argmax}}\left\langle\beta, \Gamma_{B} \beta\right\rangle
$$

$\beta_{j}=\beta \in S_{\perp},\left\langle\beta, \beta_{i}\right\rangle=0$ for $i<j\left\langle\beta, \Gamma_{B} \beta\right\rangle$ for $j=2, \ldots, c^{\prime}$;

$$
\left.\beta_{c^{\prime}+1}=\underset{\beta \in S_{\|}}{\operatorname{argmax}}\left\langle\beta, \Gamma_{\beta} \beta\right\rangle\right\rangle
$$

and

$$
\beta_{c^{\prime}+j}=\beta \in S_{\|},\left\langle\beta, \beta_{c^{\prime}+i}\right\rangle=0 \text { for } i<j\left\langle\frac{\left\langle\beta, \Gamma_{B} \beta\right\rangle}{\operatorname{argmax}\rangle} \text { for } j=2, \ldots, c^{\prime \prime},\right.
$$

where $\int \beta^{2}(v) d v=1$ for identifiability, $S_{\perp}$ is the projection of $S_{B}$ on the orthogonal complement of $S_{W}, S_{\|}$is the projection of $S_{B}$ on $S_{W}$, and $c^{\prime}$ and $c^{\prime \prime}$ are the dimension of $S_{\perp}$ and $S_{\|}$, respectively. The SLDA projections are optimal in the sense that when (a) $S_{B} \perp S_{W}$, all the projections are in $S_{\perp}$ and nearly perfect classification can be achieved; (b) $S_{B} \subseteq S_{W}$, all the projections are in $S_{\|}$and the projections are identical to those of LDA. After the projections are obtained, Naïve Bayes, support vector machines, or some conventional classifier could be applied to the projected values. Examples and detailed derivations of theoretical results can be found in the original paper of SLDA. 
Section S4. Classification results of clinical MTBC and NTM strains using PCA and PCA-DA
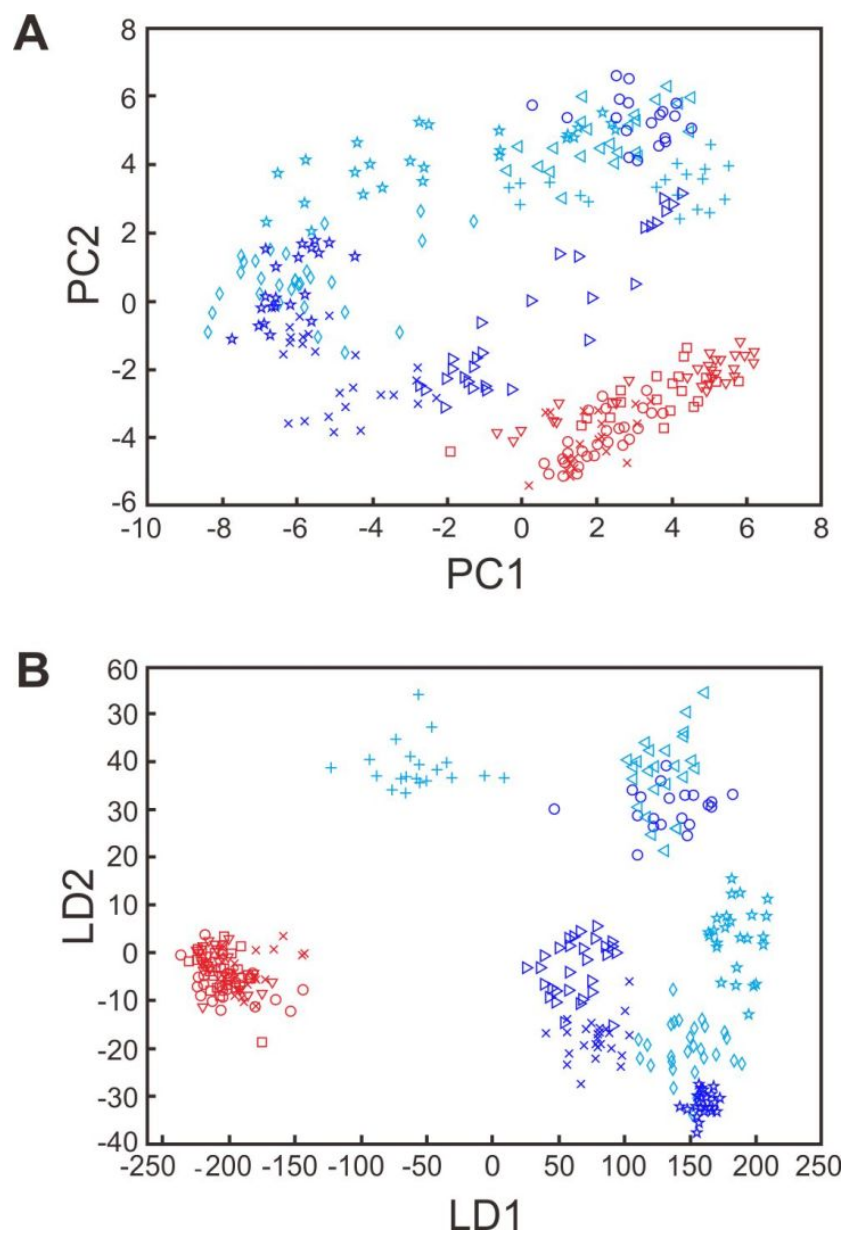

Figure S2. Projection results of (A) principal component analysis (PCA) and (B) PCA followed by linear discriminant analysis (PCA-DA) of individually acquired SERS spectra of mycobacteria: four clinical MTBC strains $(\nabla, \square, \bigcirc$ and $\times)$, clinical Mycobacterium abscessus $(O)$, clinical Mycobacterium intracelluare ( $\downarrow$ ), clinical Mycobacterium gordonae $(\triangleleft)$, clinical Mycobacterium marinum $(\bigotimes)$, clinical Mycobacterium mucogenicum (+), clinical Mycobacterium chelonae (メ), clinical Mycobacterium neoaurum ( $\triangleright)$, and clinical Mycobacterium xenopi ( Ł). Each symbol represents a measured SERS spectrum. 


\section{References}

(1) Wang, H.-H.; Liu, C.-Y.; Wu, S.-B.; Liu, N.-W.; Peng, C.-Y.; Chan, T.-S.; Hsu, C.-F.; Wang, J.K.; Wang, Y.-L. Highly Raman Enhancing Substrates Made of Ag-Nanoparticle Array with Tunable Sub-10 nm Gaps. Adv. Mater. 2006, 18, 491-495.

(2) Biring, S.; Wang, H.-H.; Wang, J.-K.; Wang, Y.-L. Light scattering from 2D arrays of monodispersed Ag-nanoparticles separated by tunable nano-gaps: spectral evolution and analytical analysis of plasmonic coupling. Opt. Exp. 2008, 16, 15312-15324.

(3) Cheng, T.-Y.; Wang, H.-H.; Chang, S. H.; Chu, J.-Y.; Lee, J.-H.; Wang, Y.-L.; Wang, J.-K. Revealing local, enhanced optical field characteristics of Au nanoparticle arrays with $10 \mathrm{~nm}$ gap using scattering-type scanning near-field optical microscopy. Phys. Chem. Chem. Phys. 2013, $15,4275-4282$.

(4) Limousin, G.; Gaudet, J.-P.; Charlet, L.; Szenknect, S.; Barthès, V.; Krimissa, M. Sorption isotherms: A review on physical bases, modeling and measurement. Appl. Geochem. 2007, 22, $249-275$.

(5) Chen, L. H.; Jiang, C. R. Sensible functional linear discriminant analysis. Comput. Stat. Data An. 2018, 126, 39-52. 